\title{
Tamed Tigers: Stabilization of Reactive Carbenes
}

\author{
Martin Albrecht ${ }^{*[a]}$
}

The behavior of tigers, and of wild cats generally, tends to be difficult to predict, let alone control. Carbenes are the chemical equivalents of wild cats, long believed to be too reactive to be isolated and handled appropriately. The reactivity of carbenes is readily appreciated by considering their electronic structure, comprising a carbon center with six electrons, four of which are involved in bonding to substituents ( $R$ and $R^{\prime}$ in Figure 1). This electron count violates

$$
\mathrm{R}_{\mathrm{R}^{\prime}}
$$

Figure 1. Generic representation of a carbene, highlighting the two electrons at carbon that are not engaged in bonding.

Lewis' famous octet rule, which is probably most rigidly observed for carbon. ${ }^{[1]}$ It is this electron deficit at carbon that accounts for the high reactivity of carbenes and the difficulties in stabilizing and studying these species.

Depending on the hybridization and the occupation of the orbitals of carbon, singlet and triplet ground states of carbenes can be distinguished. The electronic configuration is predominantly controlled by the Coulombic repulsion, the separation between the $\sigma$ and $\pi$ orbitals, and the steric and electronic effects induced by the substituents $R$ and $\mathrm{R}^{\prime}$ (the $\mathrm{R}-\mathrm{C}-\mathrm{R}^{\prime}$ angle, and inductive and mesomeric factors). ${ }^{[2]}$ Generally, $\pi$ donor heteroatoms such as nitrogen or oxygen strongly stabilize singlet ground states through destabilization of the carbene $p$ orbital, while aryl substituents reduce the singlet-triplet gap due to their lowenergy $\pi^{*}$ orbitals. Appropriate choice of

[a] Prof. M. Albrecht

Department of Chemistry

University of Fribourg, Chemin du Musée 9

$\mathrm{CH}-1700$ Fribourg (Switzerland)

Fax: $(+41) 263009738$

E-mail:martin.albrecht@unifr.ch the $\alpha$ substituents $R$ and $R^{\prime}$ therefore allows for tailoring the singlet-triplet gap, and today even carbenes which can exist as metastable intermediates in both spin states are known. ${ }^{[3]}$

The stability of carbenes as intermediates and their utility for organic transformations has been recognized, for example in the cyclopropanation of olefins with $\mathrm{CCl}_{2} \cdot{ }^{[4]}$ First progress in taming the reactivity of carbenes has been achieved in the mid-60s by using metals for stabilizing the reactive species. Fischer, in his seminal work, ${ }^{[5]}$ reported the sequential addition of a nucleophile and an electrophile to a metal carbonyl complex, which afforded a metal-bonded oxycarbene (2, Scheme 1$)$.

$$
\mathrm{L}_{5} \mathrm{M}-\mathrm{CO} \frac{\text { 1) } \mathrm{R}^{-}}{2) \mathrm{R}^{\prime+}} \quad \mathrm{L}_{5} \mathrm{M}=\underbrace{}_{2}=\underset{\mathrm{R}}{\mathrm{C}_{-}^{\prime}}
$$

Scheme 1. Oxycarbene formation within the metal coordination sphere according to Fischer's methodology.

While theoretical and spectroscopic advances provided considerable insight into the bonding situation and the reactivity of carbenes, including also the generally more reactive triplet carbenes, $^{[2,6]}$ attempts to isolate free carbenes initially had little success. Based on the stabilizing effect of $\alpha$-positioned donor atoms, Wanzlick erroneously proposed the formation of imidazolinylidenes 4 from electron-rich olefins such as entetramines $\mathbf{3}$ (Wanzlick equilibrium, Scheme 2). ${ }^{[7]}$

An unambiguous characterization of this type of carbene was unattainable until 1989, when Bertrand isolated the phosphino-silyl carbene 5 (Figure 2). ${ }^{[8]}$ Soon afterwards, Arduengo provided structural proof of free carbenes through $\mathrm{X}$-ray diffraction analyses of the thermal-

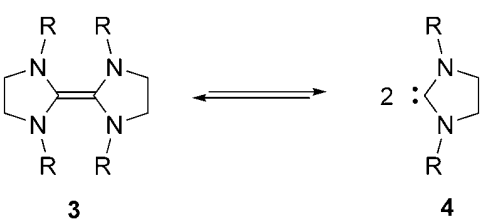

Scheme 2. The supposed Wanzlick equilibrium between enetetramines and imidazolinylidenes.

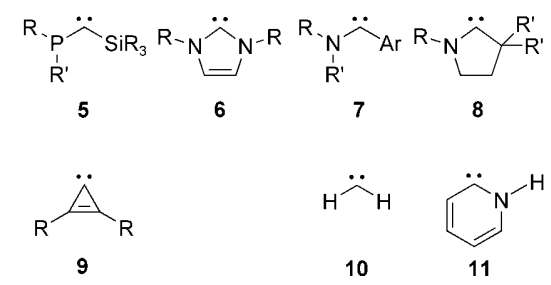

Figure 2. Selection of free carbenes that have been characterized by crystallography (5-9) or by spectroscopic methods $(10,11)$.

ly stable imidazolylidenes 6 (Figure 2). ${ }^{[9]}$ Bulky substituents, $(R=$ e.g. adamantyl, tolyl) were installed at the nitrogen atoms in order to reduce the kinetic lability of the formed carbene. This progress nicely demonstrates the potential relevance of initially unsuccessful approaches such as Wanzlick's equilibrium suggestion. Following these breakthroughs in isolating free $\mathrm{N}$-heterocyclic carbenes, a number of carbenes comprising less extensive heteroatom stabilization have been characterized crystallographically, such as stable phosphinoand amino-aryl carbenes (7), cyclic amino-alkyl carbenes (CAAC, 8) and their acyclic analogs, in particular through the pioneering work of Bertrand and coworkers. ${ }^{[10]}$ Even carbenes stabilized by remote heteroatoms, such as cyclopropylidene $9\left(R=\mathrm{NiPr}_{2}\right)$, which was previously investigated by spectroscopic tools, were isolated and structurally characterized by single-crystal X-ray diffraction. ${ }^{[11]}$

For analyzing carbenes with only limited stability, for example those substitut- 
ed by only weak $\pi$ donors, two approaches have found the widest application: $\left.{ }^{[12]} 1\right)$ matrix isolation at low temperatures in order to reduce bimolecular reactions and to decelerate decomposition reactions, and 2 ) in situ analysis at high energies, at which less stable conformers become increasingly populated. The former method has been used for the characterization of typically highly reactive triplet carbenes. ${ }^{[6]}$ While most of the carbenes, including the prototypical carbene : $\mathrm{CH}_{2}(10)$, have only limited possibilities for further intramolecular reactions-for example when isolated in a matrix - the situation is markedly different for carbenes with heteroatoms that contain labile protons (such as $R=N R^{\prime} H$, $\mathrm{OH}, \mathrm{SH}$ ) in the $\alpha$ position. For example, 2-pyridylidene $\mathbf{1 1}$ is a tautomer of pyridine and is supposed to undergo a fast intramolecular proton migration from nitrogen to carbon. Despite this reactivity, pyridylidenes were detected in the gas phase. ${ }^{[13]}$ Recently, the tautomeric equilibrium was successfully shifted to the pyridylidene side in the presence of Os and Ir, though metal coordination appeared to be essential for stabilizing the pyridylidene tautomer. ${ }^{[14]}$

Similar tautomerization into a carbene species has been proposed for aldehydes, specifically also for formaldehyde. ${ }^{[15]}$ Despite the fact that the corresponding hydroxymethylene 13 was predicted to be stable by quantum-chemical calculations, hydroxycarbenes have remained elusive. Very recently, though, Schreiner et al. reported on the successful preparation and spectroscopic characterization of hydroxymethlyene. ${ }^{[16]}$ Different synthetic approaches towards hydroxymethylene are conceivable, such as the dehydrogenation of methanol or $\mathrm{N}_{2}$ elimination from diazirines. The method chosen by the authors starts from glyoxylic acid (12, Scheme 3 ) and involves first, and quite remarkable for the preparation of a supposedly reactive inter-

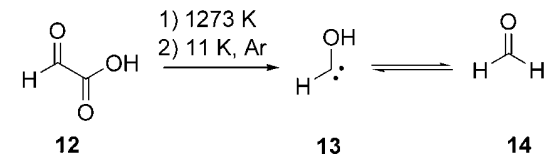

Scheme 3. Synthesis of hydroxymethylene 13 and tautomerization to formaldehyde 14 mediate, a thermally induced decarboxylation by high-vacuum flash pyrolysis followed by the trapping of the products in a solid argon matrix at $11 \mathrm{~K}$.

Careful optimization of the reaction conditions allows for the accumulation of sufficient amounts of carbene $\mathbf{1 3}$ for analyses by IR and UV/Vis spectroscopy. The assignment of the vibrational band origins is supported by quantum-chemical force field calculations, which are in excellent agreement with the observed data. However, even in the 11-20 K temperature range, rearrangement to the thermodynamically more stable formaldehyde takes place spontaneously $\left(t_{1 / 2}\right.$ is approximately $2 \mathrm{~h}$ ) and it can be significantly accelerated by irradiation at $430 \mathrm{~nm}$ into the absorbance band of the singlet carbene. While the rate of tautomerization is not particularly sensitive to temperature changes, deuterium labelling of the oxo-bound hydrogen increases the stability of the carbene significantly. The carbene :C(H)(OD) [13'] does not show any rearrangement to the corresponding monodeuterated formaldehyde. This dramatic influence cannot be rationalized by a simple kinetic isotope effect (KIE), but rather is-combined with the temperature-independent tautomerization rate of 13 - a good indication for a tunnelling mechanism.

Quantum-mechanical models support a tunnelling process. Half-lives of $t_{1 / 2}=$ $122 \mathrm{~min}$ and $t_{1 / 2}=1200$ years were calculated for 13 and its monodeuterated analog 13', respectively, thus rationalizing the observed stability of these species. ${ }^{[16]}$ Hydroxymethylene resides in a deep well on the potential energy surface. The calculated $0 \mathrm{~K}$ activation enthalpy for the formation of formaldehyde from 13 was predicted to be about $30 \mathrm{kcalmol}^{-1}$. Also, rotation about the $\mathrm{C}-\mathrm{O}$ bond and formation of the isomer with the hydrogen atoms in a cisoid position was calculated to require close to $30 \mathrm{kcal} \mathrm{mol}^{-1}$. Similar barriers were computed for a potential singlet-to-triplet intersystem crossing, both at the ground and the transition state. These high activation energies probably made it possible for hydroxymethylene to survive the drastic conditions used for its formation until trapped in the cold matrix.
According to the relationship between the activation energy $E_{\mathrm{a}}$ and the reaction rate $k$ [Arrhenius equation, Eq. (1)]:

$k=A e^{-\mathrm{E}_{\mathrm{a}} / \mathrm{RT}}$

the $30 \mathrm{kcal} \mathrm{mol}^{-1}$ activation energy calculated for the tautomerization translates to a reaction rate that is essentially zero in the cold matrix $(T=11 \mathrm{~K})$ if classical pathways are followed. For $T=1273 \mathrm{~K}$, that is, under conditions used for flash pyrolysis, and assuming an exclusively intramolecular first-order process $(A \approx$ $\left.10^{13}\right)$, the conventional tautomerization rate is extremely high, $k_{1273} \approx 7 \times 10^{7} \mathrm{~s}^{-1}$. Such a virtually instantaneous reaction may illustrate the difficulties associated with preparing large quantities of $\mathbf{1 3}$ by pyrolysis and underlines the relevance of optimized reaction conditions for the synthesis.

At room temperature the lifetime of $: \mathrm{C}(\mathrm{H})(\mathrm{OH})$ is limited by tunnelling processes (estimated thermal tautomerization rate $k_{298} \approx 10^{-9} \mathrm{~s}^{-1}$ ). Metal coordination and the formation of complexes such as 15 (Scheme 4) could be advanta-

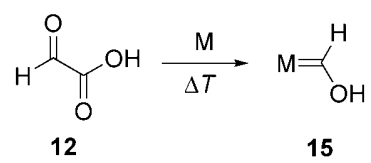

Scheme 4. Proposed metal-stabilization of carbene 13 by formation of complex 15 .

geous for suppressing tunnelling events and for accumulating synthetically useful quantities of 13. This approach may, at the same time, perhaps reduce the amount of undesired products like $\mathrm{CO}$, as observed during high-vacuum flash pyrolysis. While metal coordination should enable further characterization of this archetype of oxycarbenes, it will also allow for exploiting the application potential of hydroxymethylene and its alkylated derivatives. Applications of hydroxycarbenes should be of great interest, in particular given the enormous impact of related carbenes as ancillary ligands and reactive intermediates in metal-mediated catalysis. $^{[4,17]}$

A large variety of applications can be envisaged due to the presence of both 
$\alpha$ and $\beta$ hydrogens in a metal-bonded hydroxymethylene complex 15 . For example, carbenes such as $\mathbf{1 3}$ are expected to play a role beyond that of an ancillary ligand and they are likely to participate actively in (metal-mediated) transformations, perhaps by engaging in reversible hydrogen transfer interactions.

Unlike many $\mathrm{N}$-heterocyclic carbenes, hydroxymethylene is still far from being domesticated. Schreiner and coworkers succeeded however, in taming this chemical wild cat to such an extent that controlled reactions involving this carbene species are now conceivable. This achievement represents undoubtedly another landmark in demonstrating the potential of carbenes as components for synthesis. Along these lines, it may be interesting to study less conventional carbenes, such as 4-imidazolylidenes, that is, tautomers of the Arduengo-type carbenes 6 , and 3- and 4-pyridylidenes (i.e. the tautomers of 11). ${ }^{[18]}$ In particular, it should be instructive to learn whether these species belong to the class of carbenes or whether they are better described as zwitterionic compounds. ${ }^{[19]}$ Clearly, matrix isolation supported by sophisticated computational analyses may pave the way for achieving further progress in this area.

\section{Acknowledgements}

The author thanks Thomas Bally for fruitful discussions and the Alfred Werner
Foundation for support through an Assistant Professorship.

Keywords: carbenes - matrix isolation · metal-stabilization . spectroscopy. tautomerism

[1] G. N. Lewis, J. Am. Chem. Soc. 1916, 38, 762.

[2] M. Jones, Jr., R. A. Moss in Reactive Intermediate Chemistry (Eds.: R. A. Moss, M. S. Platz, M. Jones, Jr.), Wiley-Interscience, Hoboken, NJ, 2004, p. 273.

[3] Z. Zhu, T. Bally, L. L. Stracener, R. J. McMahon, J. Am. Chem. Soc. 1999, 121, 2863.

[4] a) W. von E. Doering, A. K. Hoffmann, J. Am Chem. Soc. 1954, 76, 6162; see also b) R. R. Schrock, G. W. Parshall, Chem. Rev. 1976, 76, 243 ; c) T. M. Trnka, R. H. Grubbs, Acc. Chem. Res. 2001, 34, 18; d) M. P. Doyle, D. C. Forbes, Chem. Rev. 1998, 98, 911.

[5] E. O. Fischer, A. Maasböl, Angew. Chem. 1964 76, 645; Angew. Chem. Int. Ed. Engl. 1964, 3, 580.

[6] H. Tomioka in Reactive Intermediate Chemistry (Eds.: R. A. Moss, M. S. Platz, M. Jones, Jr.) Wiley-Interscience, Hoboken, NJ, 2004, p. 375.

[7] a) H.-W. Wanzlick, E. Schikora, Angew. Chem 1960, 72, 494; b) D. M. Lemal, R. A. Lovald, K. I. Kavano, J. Am. Chem. Soc. 1964, 86, 2518 ; c) T. A. Taton, P. Chen, Angew. Chem. 1996, 108, 1098; Angew. Chem. Int. Ed. Engl. 1996, 35, 1011.

[8] A. Igau, A. Baceiredo, G. Trinquier, G. Bertrand, Angew. Chem. 1989, 101, 617; Angew. Chem. Int. Ed. Engl. 1989, 28, 621.

[9] A. J. Arduengo III, R. L. Harlow, M. Kline, J. Am. Chem. Soc. 1991, 113, 361.

[10] a) C. Buron, H. Gornitzka, V. Romanenko, G. Bertrand, Science 2000, 288, 834; b) S. Solé H. Gornitzka, W. W. Schoeller, D. Bourissou, G. Bertrand, Science 2001, 292, 1901; c) V. LavalIo, J. Mafhouz, Y. Canac, B. Donnadieu, W. W. Schoeller, G. Bertrand, J. Am. Chem. Soc.
2004, 126, 8670; d) V. Lavallo, Y. Ishida, B. Donnadieu, G. Bertrand, Angew. Chem. 2005, 117, 5851; Angew. Chem. Int. Ed. 2005, 44, 5705.

[11] a) H. P. Reisenauer, G. Maier, A. Riemann, R. W. Hoffmann, Angew. Chem. 1984, 96, 596; Angew. Chem. Int. Ed. Engl. 1984, 23, 641; b) V. Lavallo, Y. Canac, B. Donnadieu, W. W. Schoeller, G. Bertrand, Science 2006, 312, 722.

[12] For an alternative approach using molecular encapsulation, see: a) R. Warmuth, Eur. J. Org. Chem. 2001, 423; b) X. Liu, G. Chu, R. A. Moss, R. R. Sauers, R. Warmuth, Angew. Chem. 2005, 117, 2030; Angew. Chem. Int. Ed. 2005, 44, 1994.

[13] a) P. Dyson, D. L. Hammick, J. Chem. Soc. 1937, 1724; b) D. Lavorato, J. K. Terlouw, T. K. Dargel, W. Koch, G. A. McGibbon, H. Schwarz, J. Am. Chem. Soc. 1996, 118, 11898.

[14] a) M. A. Esteruelas, F. J. Fernandez-Alvarez, E. Oñate, J. Am. Chem. Soc. 2006, 128, 13044; b) E. Alvarez, S. Conejero, M. Paneque, A. Petronilho, M. L. Poveda, O. Serrano, E. Carmona, J. Am. Chem. Soc. 2006, 128, 13060.

[15] C.-F. Pau, W. J. Hehre, J. Phys. Chem. 1982, 86, 1252.

[16] P. R. Schreiner, H. P. Reisenauer, F. C. Pickard IV, A. C. Simmonett, W. D. Allen, E. Mátyus, A. G. Császár, Nature 2008, 453, 906.

[17] a) H. M. L. Davies. R. E. J. Beckwith, Chem. Rev. 2003, 103, 2861; b) F. E. Hahn, M. C. Jahnke, Angew. Chem. 2008, 120, 3166; Angew. Chem. Int. Ed. 2008, 47, 3122.

[18] a) M. Heckenroth, E. Kluser, A. Neels, M. Albrecht, Angew. Chem. 2007, 119, 6409; Angew. Chem. Int. Ed. 2007, 46, 6293; b) H. G. Raubenheimer, S.J. Cronje, Dalton Trans. 2008, 1265; c) M. Albrecht, Chem. Commun. 2008, 3601.

[19] C.P. Newman, G. J. Clarkson, N. W. Alcock, J. P. Rourke, Dalton Trans. 2006, 3321. 